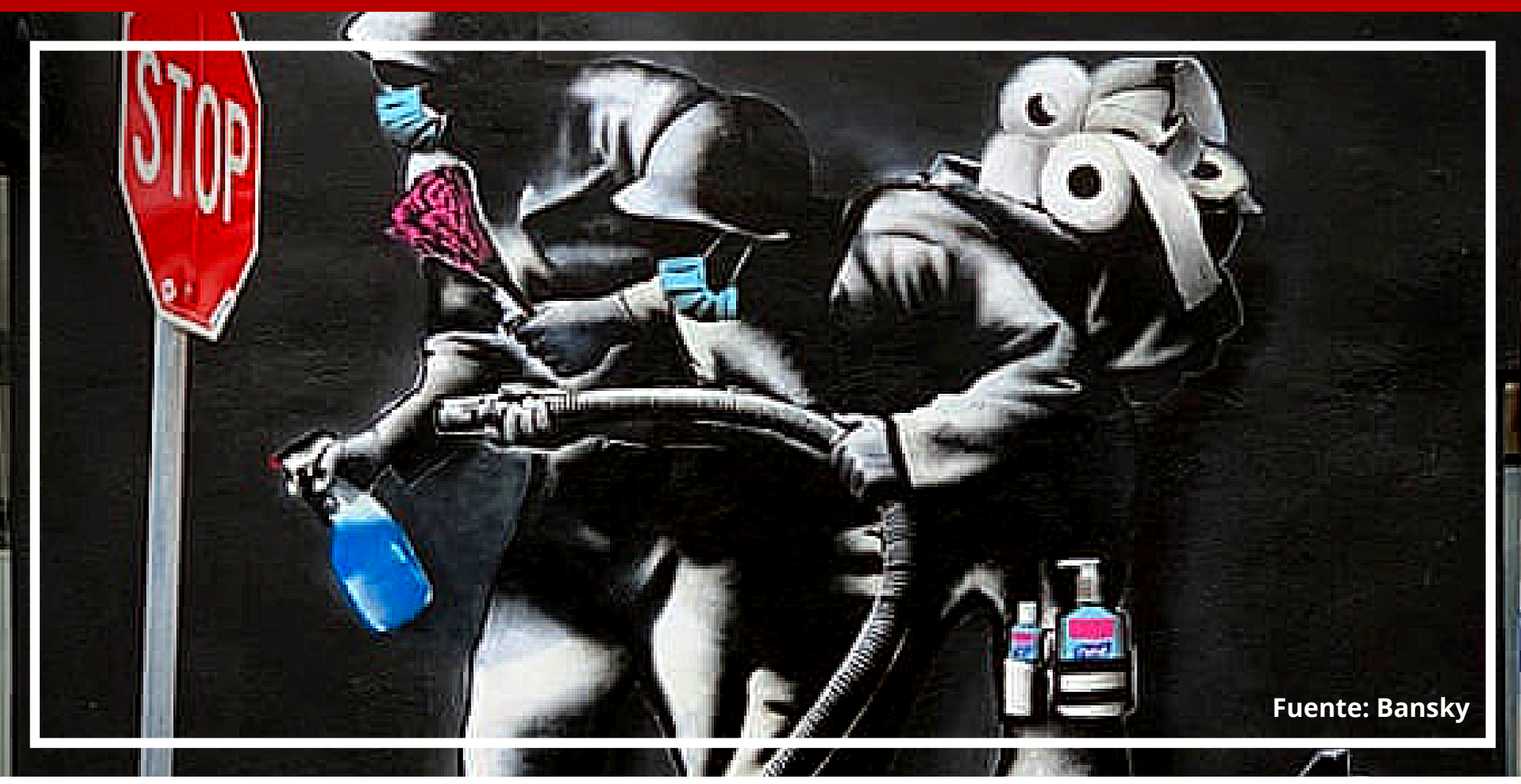

\title{
Ética y Pandemia ¿Es la cuarentena una medida moral?
}

\section{doi: $10.52749 /$ rh.v1i1.4}

\section{PIERO GAYOZZO}

iD https://orcid.org/0000-0002-5112-5431

Coordinador General de la Sociedad Secular Humanista del Perú (SSH). Fundador y Sub Director del Instituto de Estudios Transhumanistas (IET). Miembro de la Asociación Peruana de Comunicadores y Periodistas Científicos (APCIENCIA) y de la Asociación Peruana de Ateos APERAT. Llevó estudios de ingeniería industrial en la Universidad de Lima. Miembro del Consejo del Fondo Editorial de la Sociedad Secular Humanista del Perú.

$\checkmark$ pgayozzo@ssh.org.pe 0 @pgayozzo

Resumen. El pasado 2019, un nuevo coronavirus emergió en la localidad china de Wuhan. Meses después, la Organización Mundial de la Salud declaró su brote como pandemia. Tras ello, múltiples estrategias han sido desplegadas para lidiar con su propagación, una de ellas es la cuarentena. Así, con la pandemia de SARS-CoV-2 se ha vuelto a considerar el debate sobre si es moral o no dicha medida. En este artículo se procurará responder a la pregunta desde un enfoque humanista secular y aplicando el método de bioética empírica denominado contribuciones empíricas paso a paso.

Palabras clave: coronavirus, ética, humanismo secular, pandemia, SARS-CoV-2.

Cómo citar este artículo:

Gayozzo, P. (2021). Ética y pandemia. ¿Es la cuarentena una medida moral? Revista Humanista, 1(1), 23-28. https://doi.org/10.52749/rh.vlil.4

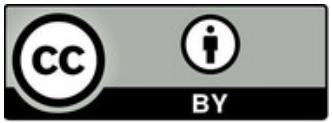

Esta obra está bajo licencia internacional

Creative Commons 4.0 Reconocimiento 4.0. 


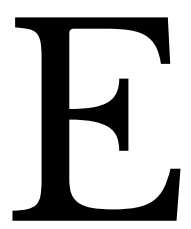

131 de diciembre del 2019, la Oficina de la Organización Mundial de la Salud (OMS o WHO, por sus siglas en inglés) en China publicó el primer reporte emitido por las autoridades chinas de la localidad de Wuhan sobre la emergencia de un nuevo tipo de neumonía (WHO, 2020). El 07 de febrero se publicó un artículo sobre las similitudes de la composición genética del patógeno que lo causaba: un nuevo coronavirus $(\mathrm{Wu}, \mathrm{y}$ otros, 2020) nombrado como SARS-CoV-2 al mes siguiente (ICTV, 2020). El 11 de marzo del 2020, la OMS declaró la situación del nuevo coronavirus como pandemia (WHO, 2020). Hasta la fecha, el SARS-CoV-2 ha matado a 2 173965 personas (Johns Hopkins University, 2021).

A lo largo de esta pandemia ha habido múltiples cuestionamientos y discrepancias con las indicaciones de la OMS y las políticas nacionales. Muchas de estas involucran teorías de conspiración (Kearne et al., 2020), así como curas milagrosas y pseudoterapias (Gayozzo, 2020). No obstante, también se han esgrimido cuestionamientos sobre el impacto económico de una medida tan radical como la cuarentena (Milei, 2020). En el siguiente artículo intentaremos responder a si optar por una cuarentena es moral o no desde un enfoque realista y humanista.

¿Qué es una cuarentena? Según los Centros para el Control y la Prevención de Enfermedades (en adelante CDC, por sus siglas en inglés), la cuarentena es una estrategia de protección pública que separa y restringe el movimiento de personas que fueron expuestas a enfermedades contagiosas con el objetivo de determinar si llegan a desarrollar la enfermedad (CDC, 2017). Como tal, implica reducir la libertad para disminuir la posibilidad de contagio y, por consiguiente, mantener el bienestar de las personas.

Los conceptos de libertad y de bienestar son claves para el desarrollo de nuestro argumento, por ello, esbozaremos las definiciones a las que recurriremos.

Definir la libertad como la capacidad de decidir espontáneamente trae consigo el conflicto entre dos enfoques filosóficos: el libertarianismo y el determinismo. Desde un enfoque naturalista, el libre albedrío es una ilusión por cuanto es imposible desvincular un evento de causas anteriores, ya sean biológicas o contextuales (Harris, 2012). En este artículo nos acogeremos a esta visión sin desmedro de que una persona sea responsable de sus actos. Tal cual ha descrito Pinker, la falacia de la eliminación de responsabilidad alguna de un individuo sobre sus actos como consecuencia de la inexistencia del libre albedrío se sustenta en una confusión entre explicación y excusación. Además, la validez del criterio de responsabilidad dependerá de cuál sea el valor y la función que le asignemos, es decir, mantenerla como medio para disuadir el comportamiento dañino (Pinker, 2008).

Definiremos la libertad como la capacidad de hacer algo sin ser limitado por factores naturales, y de obtener los medios para ello. Así, aunque esta facultad nos sea ilusoria, no existe razón para evadir responsabilidad sobre nuestros actos.

Por su parte, el concepto de bienestar, como lo describe Harris, es de por sí complejo. Por ello en el siguiente artículo emplearemos el Índice de Bienestar Personal de Cummins et al. (Cummins et al., 2003) no solo por la solidez con la que representa el bienestar, sino porque, tal cual indica Alfaro et al. (2014) el índice ha sido utilizado por más de 100 investigadores en 50 países y ha mostrado un buen rendimiento de validez, fiabilidad y sensibilidad en poblaciones de adultos de muchos países.

Según aquella herramienta psicométrica, el bienestar se vincula con siete dimensiones: estándar de vida, salud, logros en la vida, relaciones personales, seguridad personal, vinculación con la comunidad y seguridad futura.

\section{Método}

A partir de la visión cientificista de la ética propuesta por Harris (2010), Kurtz (2007), Purdy (2007) y Bunge (1989), se procurará un breve análisis del problema moral en cuestión recurriendo al conocimiento científico disponible.

En bioética empírica se conoce a este método como "contribuciones empíricas paso a paso" (Davies et al., 2015). Según Borry et al. (2004), este método recoge las contribuciones empíricas de otras disciplinas y las integra en varias etapas de la investigación bioética: desde la descripción del objeto moral y la evaluación del problema moral hasta el proceso de toma de 
decisiones. Contrario a Davies et al. para quienes este método puede enfrentar la limitante de emplear la data para avalar una teoría moral, consideramos que la revisión de dicha teoría debe realizarse cuando se posea mayor evidencia empírica, que sería parte de una investigación posterior.

Sobre la dicotomía hecho/valor, no serán contempladas como factores opuestos, sino íntimamente ligadas, por consiguiente, como factores indispensables del proceso cíclico de revisión entre técnicas descriptivas y normativas que esta metodología propone (Cribb \& Owens, 2017).

Para ello, el sistema moral al que nos adscribiremos será el humanista secular. Este se caracteriza porque, a diferencia de deontológico, es consecuencialista - sus normas se construyen en función de las consecuencias de las acciones-, realista - se adecua a diferentes contextos-, secular - no recurre a intervenciones ni entes divinos-, científico - recurre al conocimiento científico para valorar lo bueno y lo justo-, sistémico ni individualista ni holista-, así como universalista y racional -en lugar de subjetivo y dogmático- (Bunge, 1989).

Semejante sistema moral se edifica a partir de un axioma, el cual deberá probar su eficacia para seguir siendo válido. El axioma propuesto por Bunge es el de "gozar de la vida y ayudar a los demás a vivir una vida digna”. En el siguiente artículo consideraremos que este axioma permite cruzar la brecha hecho/valor, pues brinda la suficiente justificación moral a los hechos para que estos se puedan articular como normas morales.

Dicho sistema moral plantea un esquema de valores en función de las necesidades y deseos legítimos de toda persona, los primeros vinculados a la supervivencia y los segundos al bienestar. De ahí que podamos ordenar y otorgar cierto nivel de prioridad a los criterios del índice de bienestar priorizando los relativos a la salud por sobre los sociales.

El dilema reside en si la decisión de aplicar una cuarentena para la pandemia de covid-19 es moral o no, es decir, si es buena por la cantidad de razones favorables que se pueden identificar (Savulescu, 2002) o inmoral si es razonablemente injustificable. Separaremos entonces dicho episodio por facetas: i. etapa temprana o pre-pandémica, tiempo compren- dido desde la aparición del virus hasta los primeros días en que su propagación se catalogó como pandemia, y ii. el desarrollo de la pandemia en sí misma. La razón para esta separación es el responder si es moral la decisión de ejecutar una cuarentena, simulando un tiempo en el cual no se han desatado todas sus consecuencias, y no responder sobre si fue moral dictar una cuarentena, pregunta que se debe responder con un análisis de las consecuencias reales del evento.

\section{Discusión}

Situados en un escenario pre-pandémico nos preguntamos ¿debe optarse por ejecutar una cuarentena? La cuarentena, como se vio, reduce la libertad de movimiento para mantener el bienestar personal y comunitario. En una sociedad con alto flujo de capitales, la reducción de la libertad de movimiento tendría como consecuencia paralizar las actividades económicas. Al hacerlo, solo se podría mantener la salud de la que las personas ya gozan hasta ese momento. A ello cabe agregar que, dependiendo de la duración del confinamiento, la salud psicológica de las personas podría también verse afectada. Evaluemos lo que la evidencia científica nos puede decir de cada escenario.

La paralización de las actividades económicas nos situaría - tal cual se ha evaluado en los casos del MERS y del SARS - ante una serie de problemas similares a los de una recesión económica, entre ellos el cierre de negocios, la reducción de puestos laborales (Lee \& Cho, 2016) y la disminución de la tasa de crecimiento nacional (Ceylan \& Ozkan, 2020). Es un escenario que impactaría en la capacidad de compra de alimentos de muchas familias. A su vez, el cierre de los espacios y negocios de esparcimiento podría afectar la salud psicológica de muchos, al igual que lo haría la consecuente acumulación de deudas. De acuerdo con nuestro esquema de valores, los principales problemas a evaluar son los relativos a la supervivencia y salud: potencial falta de alimentos y cuadros perjudiciales de salud mental.

¿Cuánto tiempo debe pasar para afectar mortalmente a una persona por falta de alimentos? Existen reportes de personas que fueron sometidas a tortura o privación de alimentos voluntaria que han sobrevivido entre 
20 y 40 días, pero el promedio usual de supervivencia es de hasta tres semanas (Lieberson, 2004) (Kottusch et al., 2009). Si bien estos casos son extremos, podemos contemplar los efectos de la privación de alimentos en el organismo para estados de inanición en el caso de personas con trastornos alimenticios previos y de malnutrición o deficiencia de nutrientes. Los efectos inmediatos son pérdida de peso y masa corporal, reducción del funcionamiento muscular, deficiencia cardíaca, alteraciones hormonales y del sistema digestivo y efectos negativos sobre el sistema inmunológico (Saunders, 2010), así como efectos conductuales y cognitivos obtenidos del famoso experimento Keys (1944) en el que se halló que los individuos sometidos a una reducción de la ingesta de calorías por 6 meses sufrieron alteración de la concentración, de la comprensión, del juicio y del sentido del humor, depresión, ansiedad, aislamiento, irritabilidad y apatía, entre otros.

Por otro lado, en el año 2003 durante el brote de SARS en la ciudad de Toronto, 15000 personas fueron puestas en cuarentena. Tras sus respectivos tiempos de confinamiento, 129 personas respondieron una encuesta cuyo resultado arrojó que aquellos con ingresos anuales menores a 40 mil dólares canadienses tuvieron mayor presencia de síntomas de estrés post-traumático y depresión (Hawryluck et al., 2004). Resultados similares fueron obtenidos de la evaluación de 1692 personas colocadas en cuarentena durante el brote de MERS en Corea del Sur, de las cuales el 7.6\% de ellas mostró síntomas de ansiedad y el $16.6 \%$ mostró ira (Jeong et al., 2016). Es evidente la afección psicológica de las personas en cuarentena, pero estos no son problemas que puedan tornarse mortales.

Evaluemos ahora el escenario en el cual la población es expuesta al patógeno. La primera pregunta que surge es ¿qué tan mortal es la enfermedad? Si es un patógeno nuevo hemos de suponer que no se conoce al detalle su naturaleza. Por lo tanto, su tasa de mortalidad es incierta. En vista de que algunas enfermedades pueden complicarse en conjunto, la mortalidad del nuevo patógeno puede aumentar. Eso quiere decir que exponer a las personas sanas y no sanas a un nuevo patógeno con capacidades inciertas quebraría o pondría en riesgo el pilar principal sobre el que se erige nuestro sistema de valores: aquellos concernientes a la salud y a la supervivencia. ¿Puede existir bienestar de algún tipo con una salud afectada o en personas muertas? Obviamente no.

Ya que nuestro sistema moral es secular, por ende, naturalista, se asume que la vida que poseemos es la única vida que vamos a tener. De ahí que sea imperativo cuidarnos y cuidar a los demás, esta estrategia no solo garantizaría nuestra supervivencia como especie, sino que compatibiliza con la máxima humanista propuesta - "Goza de la vida y ayuda a los demás a vivir dignamente"-, y con las dimensiones de salud, seguridad personal y seguridad futura del índice de bienestar de Cummins et al. (2003).

Como el estrés y una alimentación limitada no son factores de muerte inmediatos, la cuarentena se tornaría una solución moral, pues pese a tener un costo directo sobre las otras dimensiones del índice de bienestar, específicamente sobre las relaciones personales, la vinculación con la comunidad, los logros en la vida y el estándar de vida, garantiza la supervivencia de la mayor población posible.

Lo cierto es que, una vez resuelto el problema moral, las posibles consecuencias de la cuarentena se tornan problemas técnicos más que problemas morales. Para mitigar el impacto negativo y sus repercusiones en la salud de la población, una decisión más coherente - sobre todo si procuramos apegamos a un sistema moral realista- debe mantenerse sujeta a la disposición de cooperación y a las soluciones que pueden tomarse en conjunto entre los actores comunitarios, el sector privado y el estatal. En vista de que nuestro sistema moral también es consecuencialista, indicamos que es importante contemplar las cuatro razones para justificar una cuarentena de Upshur (2003):

1.La amenaza de contagio de persona a persona es visible. Esto se determina una vez detectado el patógeno, evaluado su alcance y efectos inmediatos.

2.Principio de proporcionalidad. Antes de tomar medidas restrictivas o sancionadoras, debe primar la información y la concientización. Esta medida podría reducir parte del estrés causado por el temor a un patógeno desconocido. A su vez, basados en el sentido de libertad expuesto anteriormente, una vez informada la ciuda- 
danía quien se muestre contrario a estas debe hacerse responsable por las sanciones establecidas.

3.Reciprocidad sostenida. La sociedad deberá velar por apoyar en las obligaciones a los ciudadanos en cuarentena. Para lidiar con las carencias económicas, alimenticias y sus problemas psicológicos derivados, así como los de salud pre-existentes, la comunidad debe hacer lo posible por trazar soluciones que garanticen el bienestar de la mayor cantidad de personas durante el tiempo en que serán recluidas.

4.Principio de transparencia. Las autoridades deben comunicar las razones de sus acciones. Los problemas de comunicación son en su mayoría técnicos, de ahí que sea un imperativo trazar estrategias de comunicación eficientes y que hagan uso de la mayor cantidad de canales de manera sistemática.

Tal cual evaluaron Brooks et al. (2020) los estresores de una cuarentena pueden mitigarse si se ejecuta por un periodo de tiempo corto, se mantiene informada a la gente y se entregan provisiones adecuadas. De misma forma, sería conveniente que las autoridades adopten estrategias postcuarentena para lidiar con los estragos de salud, económicos y psicológicos.

\section{Referencias}

Alfaro, J., Castellá Sarriera, J., Casas, F., Valdenegro, B., \& Oyarzún,

D. (2014). Adaptación del Índice de Bienestar Personal para adolescentes en lengua española y portuguesa. Universitars $\begin{array}{lll}\text { Psychologica, 13(1), } & \text { 1-25. }\end{array}$ https://doi.org/10.11144/Javeriana.UPSY13-1.aibp

Borry, P., Schotsmans, P., \& Dierickx, K. (2004). What is the role of empirical research in bioethical reflection and decision-making? An ethical analysis. Medicine Health Care and Philosophy, 7(1), 41-53.

https://link.springer.com/content/pdf/10.1023/B:MHEP.000002184 4.57115.9d.pdf

Brooks, S. K., Webster, R. K., Smith, L. E., Woodland, L., Wessely, S., Greenberg, N., \& Rubin, G. J. (2020). The psychological impact of quarantine and how to reduce it: rapid review of the evidence. The Lancet, 1-9. https://doi.org/10.1016/S0140$\underline{6736}(\underline{20}) \underline{30460-8}$

Bunge, M. (1989). Treatise on Basic Philosophy (Vol. 8). MTP Press.

CDC. (29 de Septiembre de 2017). Quarantine and Isolation.

Centers for Disease Control and Preevention: https://www.cdc.gov/quarantine/index.html

Ceylan, R. F., \& Ozkan, B. (2020). The economic effects of epidemics: from SARS and MERS to COVID-19. Research Journal in Advanced Humanities, 1(2), 21-29. https://royalliteglobal.com/advanced-humanities/article/view/132
Sin embargo, en vista de los efectos de una cuarentena general, es prioritario tomar una decisión rápida sobre un patógeno y considerar inicialmente el aislamiento de los pacientes sintomáticos. $\mathrm{Si}$ pese a las medidas de aislamiento aumentan los casos de contagio y la transmisión del patógeno es alto, se debería evaluar la aplicación de una cuarentena, sobre todo por haberse calculado su posible efectividad (Day et al., 2006).

\section{Conclusiones}

De por sí una cuarentena será una medida moral (justificada) pues antepone la salvaguarda de la mayor cantidad de vidas posibles. Para aumentar sus resultados positivos existen una serie de factores a ser contemplados por los actores sociales. Naturalmente, es importante indicar que, si bien optar por una cuarentena puede ser una decisión moral, son múltiples los factores técnicos que intervienen para que esta pueda ser fructífera. Desde un enfoque humanista y realista, una cuarentena será moral si prevé soluciones para los problemas psicológicos y salud que puedan surgir en el tiempo que se extienda, así como si las autoridades pueden, por lo menos, proponer estrategias viables para lidiar con las consecuencias post-cuarentena.
Cribb, A., \& Owens, J. (2017). Embracing Diversity in Empirical Bioethics. En J. Ives, M. Dunn, \& A. Cribb. Empirical Bioethics. Theoretical and Practical Perspectives (pp. 103-122). Cambridge University Press.

Cummins, R. A., Eckersley, R., Pallant, J., Van Vugt, J., \& Misajon, R. (2003). Developing a national index of subjective wellbeing: The Australian Unity Wellbeing Index. Social Indicators Research, 159-190.

Davies, R., Ives, J., \& Dunn, M. (2015). A systematic review of empirical bioethics methodologies. BMC Medical Ethics, https://doi.org/10.1186/s12910-015-0010-3.

Day, T., Park, A., Madras, N., Gumel, A., \& Wu, J. (2006). When Is Quarantine a Useful Control Strategy for Emerging Infectious Diseases? American Journal of Epidemiology, 163(5), 479-485. https://doi.org/10.1093/aje/kwjo56

Gayozzo, P. (2020). Pandemia: Coronavirus y virus mentales. NeoSkepsis.

Harris, S. (2010). The Moral Landscape: How Science Can Determine Human Values. Free Press.

Harris, S. (2012). Free Will. Free Press.

Hawryluck, L., Gold, W. L., Robinson, S., Pogorski, S., Galea, S., \& Styra, R. (2004). SARS Control and Psychological Effects of Quarantine, Toronto, Canada. Emerging Infectious Diseases, 
1206-1212. https://doi.org/10.3201/eid1007.030703

Jeong, H., Woo Yim, H., Song, Y.-J., Ki, M., Min, J.-A., Cho, J., \& Chae, J.-H. (2016). Mental health status of the quarantined people due to Middle East Respiratory Syndrome (MERS). Epidemiology and Health, 38. https://doi.org/10.4178/epih.e2016048

Johns Hopkins University. (28 de Enero de 2021). COVID-19 Dashboard by the Center for Systems Science and Engineering (CSSE) at Johns Hopkins University (JHU). Coronavirus Research Center: https://coronavirus.jhu.edu/map.html

Kearney, M. D., Chiang, S. C., \& Massey, P. C. (2020). The Twitter origins and evolution of the COVID-19 "plandemic" conspiracy theory. Harvard Kennedy School (HKS) Misinformation Review. https://doi.org/10.37016/mr-2020-42

Keys, A. (1944). Effects of Starvation. Minnesota: University of Minnesota Starvation http://www.rhondahelp.com/sites/default/files/Effects\%20of\%20S tarvation $\% 20 \%$ E2\%80\%93\%20University\%20of\%20Minnesota $\%$ 20Starvation\%20Study 1.pdf

Kottusch, P., Tillmann, M., \& Püschel, K. (2009). Survival time without

food and drink. Archiv für Kriminologie, 224, 184-191.

Kurtz, P. (2007). What is the Relationship among Science, Reason and Ethics? En P. Kurtz, \& D. Koepsell, Science and Ethics. Can Science Help Us Make Wise Moral Judgments? (págs. 11-26). Prometheus Books.

Lee, A., \& Cho, J. (2016). The impact of epidemics on labor market: identifying victims of the Middle East Respiratory Syndrome in the Korean labor market. International Journal for Equity in Health, 15(1), 196. https://doi.org/10.1186/s12939-016-0483-9

Lieberson, A. D. (08 de Noviembre de 2004). How Long Can a Person Survive without Food? Scientific American. https://www.scientificamerican.com/article/how-long-can-aperson-survive-without-food/

Milei, J. (2020). Pandemia y Socialismo: La cuarentena como delito de lesa humanidad [Video]. YouTube: https://www.youtube.com/watch?

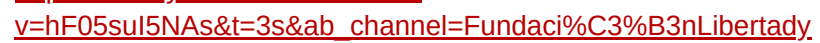
Progreso
Pinker, S. (2008). The Fear of Determinism. En J. Baer, J. C. Kaufman, \& R. F. Baumeister, Are We Free? Psychology and Free Will (págs. 311-324). Oxford University Press.

Purdy, L. (2007). The Science of Ethics. En P. Kurtz, \& D. Koepsell, Science and Ethics. Can Science Help Us Make Wise Moral Judgments? (págs. 266-273). Prometheus Book.

Saunders, J. \&. (2010). Malnutrition: causes and consequences. Clinical medicine, 10(6), 624-627. https://doi.org/ 10.7861/clinmedicine.10-6-624

Savulescu, J. (2002). Abortion, embryo destruction and the future of value argument. Journal of Medical Ethics, 133-135. https://doi.org/10.1136/jme.28.3.133

Upshur, R. (2003). The Ethics of Quarantine. Virtual Mentor, 5(11), 393-395.https://doi.org/10.1001/virtualmentor.2003.5.11.msoc10311.

WHO. (2020). Timeline: WHO's COVID-19 response. World Health Organization: https://www.who.int/emergencies/diseases/novel-coronavirus2019/interactive-timeline? gclid=Cj0KCQjw5eX7BRDQARISAMhYLP9PX4FTTWXYgdQ2lo6 CpkAkjSNw7FdPTQWPpkxrz4nA0zR4dUjow98aAg1cEALw wcB \#!

WHO. (11 de Marzo de 2020). WHO Director-General's opening remarks at the media briefing on COVID-19 - 11 March 2020. WHO: https://www.who.int/dg/speeches/detail/who-directorgeneral-s-opening-remarks-at-the-media-briefing-on-covid-19--11-march-2020

Wu, A., Peng, Y., Huang, B., Tan, W., Cheng, G., \& Jiang, T. (2020). Genome composition and divergence of the Novel Coronavirus (2019-nCoV) Originating in China. Cell Host \& Microbe, P325328. 